\title{
Response of a polymer network to the motion of a rigid sphere
}

\author{
Haim Diamant \\ Raymond \& Beverly Sackler School of Chemistry, Tel Aviv University, Tel Aviv 6997801, Israel
}

Received: date / Revised version: date

\begin{abstract}
In view of recent microrheology experiments we re-examine the problem of a rigid sphere oscillating inside a dilute polymer network. The network and its solvent are treated using the two-fluid model. We show that the dynamics of the medium can be decomposed into two independent incompressible flows. The first, dominant at large distances and obeying the Stokes equation, corresponds to the collective flow of the two components as a whole. The other, governing the dynamics over an intermediate range of distances and following the Brinkman equation, describes the flow of the network and solvent relative to one another. The crossover between these two regions occurs at a dynamic length scale which is much larger than the network's mesh size. The analysis focuses on the spatial structure of the medium's response and the role played by the dynamic crossover length. We examine different boundary conditions at the sphere surface. The large-distance collective flow is shown to be independent of boundary conditions and network compressibility, establishing the robustness of two-point microrheology at large separations. The boundary conditions that fit the experimental results for inert spheres in entangled F-actin networks are those of a free network, which does not interact directly with the sphere. Closed-form expressions and scaling relations are derived, allowing for the extraction of material parameters from a combination of one- and two-point microrheology. We discuss a basic deficiency of the two-fluid model and a way to bypass it when analyzing microrheological data.
\end{abstract}

PACS. 47.57.Qk Rheology of complex fluids - 87.19.rh Fluid transport and rheology in biological physics

\section{Introduction}

In the past two decades the technique of microrheology has been used to characterize the dynamic response of soft and biological matter [1. In one-point microrheology [2] the viscoelastic moduli of the material are inferred from the displacements of a tracer particle in response to an external force (active microrheology), or its displacement autocorrelations under thermal fluctuations (passive microrheology). Such measurements rely on a generalized Stokes relation (GSR, active) or generalized StokesEinstein relation (GSER, passive), asserting that the particle's response has the same form as in a viscous fluid, with the fluid's shear viscosity $\eta$ replaced by $\eta_{\mathrm{b}}(\omega)=$ $G_{\mathrm{b}}(\omega) /(i \omega)$. Here $G_{\mathrm{b}}(\omega)$ is the frequency-dependent complex shear modulus of the bulk material. Subsequently, two-point microrheology was introduced as well 3 . In this technique the moduli are deduced from the displacements of one particle in response to a force exerted on another (active), or the displacement cross-correlations of fluctuating particle pairs (passive), as a function of their mutual distance. The two-point measurements are based on a "generalized Oseen tensor", assuming that the spatial response at large distances is the same as in a viscous fluid, with the aforementioned replacement. Although it is considered more reliable than the one-point technique, twopoint microrheology has not been used as widely, mainly because of the difficulty to accumulate enough statistics for particle pairs at each given separation.

When results from one-point microrheology are compared with macrorheology and two-point microrheology, discrepancies are found for various materials $[3,4,5,6,7$, 8. One-point measurements commonly yield much smaller moduli than the other two techniques. The disagreement has been attributed to differences between the particle's immediate environment and the bulk material, leading to deviations from the GSR/GSER. Indeed, one-point measurements were found to be sensitive to the surface chemistry of the tracer particle [4,8, whereas two-point measurements were not 8 . Theoretical attempts to account for such local effects have included modifying the boundary conditions at the particle surface between no slip, partial slip, and full slip [7, 9], and the introduction of a shell of different viscoelastic properties surrounding the particle [10,11.

A recent study, applying two-point microrheology to entangled F-actin networks [12,13, revealed a wide range of distances, intermediate between the microscopic scale (the network's mesh size $\xi$ ) and the macroscopic (asymptotically large) one, in which the dynamic pair correlations were qualitatively different from those at larger distances. This intermediate behavior ended at a distinct dynamic length, $\ell_{\mathrm{c}}$, much larger than $\xi$, which marked the crossover to the macroscopic response. Theoretical arguments given 
in ref. 12 showed that the intermediate response was fundamentally different from the macroscopic one, in that it was related to mass transport, rather than momentum transport, of the fluid. As we shall see below, the intermediate region corresponds to the relative motion of the material's two components (network and solvent), whereas in the asymptotic region the material moves collectively, as a whole. The application of these ideas to a combination of one- and two-point measurements gives rise to an extension of microrheology, allowing for better characterization of complex fluids (e.g., extracting their correlation length) [12,13.

In the present article we elaborate on, and extend, the theoretical results briefly presented in ref. 12. We begin in sect. 2 by defining the problem, which is the analogue of Stokes' problem for the motion of a rigid sphere, with the viscous fluid replaced by a two-fluid medium [14,15, 16, 17, 18, 19. An approximate treatment of the dynamics of a bead embedded in such a medium was presented by Levine and Lubensky [18. The exact solution of the Stokes-analogous problem has already been derived by $\mathrm{Fu}$ et al. 9. In sect. 3 we solve it again from a slightly different perspective, providing additional physical insight. We derive the general solution and extract from it several general properties, which are independent of boundary conditions. In sect. 4 we present the particular solutions for three different sets of boundary conditions. The first two, corresponding to no slip and full slip of the network over the sphere surface, were treated in ref. [9] as limiting cases of a general slip condition. For these cases the present analysis offers expressions of different experimental utility. The third set of boundary conditions, which describes a network having no direct interaction with the sphere, has not been treated before. The properties of this particular solution are substantially different from the other two. We show that they match the experimental observations for entangled $\mathrm{F}$-actin networks. In sect. 4 we also derive scaling relations, which can facilitate the analysis of experimental data and the extraction of material parameters from them. Section [5 discusses in detail the results and their implications.

The analyses in refs. 9, 10, 11, 18, focused on the response of the sphere as a function of frequency. We emphasize the spatial response of the medium, its relation to basic conservation laws, and its dependencies on distance, sphere radius, and the material's characteristic lengths. We highlight, in particular, the key role played by the dynamic crossover length $\ell_{\mathrm{c}}$.

\section{The problem}

Figure 1 shows a schematic view of the system. A rigid sphere of radius $a$ moves with velocity $U$ inside a polymer network. As done in earlier studies, we use the two-fluid model [14, 15, 16, 17, 18, 19 to describe the medium. The main advantage of this model is that it is sufficiently simple to be treated analytically while delivering the key features of a complex fluid - emergent correlation length $\xi$

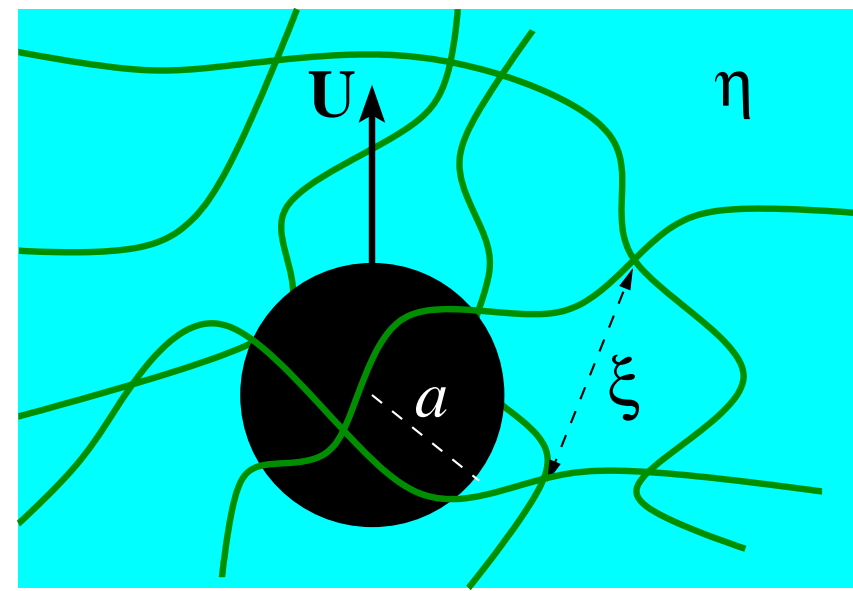

Fig. 1. Schematic view of the system.

and bulk viscoelastic modulus $G_{\mathrm{b}}(\omega)$. Its main disadvantage is that it is a continuous, linear, hydrodynamic model, neglecting effects of thermal fluctuations, small-scale heterogeneities, and nonlinear advection. An additional fundamental shortcoming of the model will be discussed in sect. 5 .

In the presentation below all fields are position- and time-dependent, and we Fourier-transform them from the time to the frequency domain,

$f(\mathbf{r}, t) \rightarrow f(\mathbf{r}, \omega)=\int_{-\infty}^{\infty} \mathrm{d} t e^{-i \omega t} f(\mathbf{r}, t)$.

The model has two components. The first is a (visco)elastic network, having a volume fraction field $\phi_{u}(\mathbf{r}, \omega)$, displacement field $\mathbf{u}(\mathbf{r}, \omega)$, and the stress tensor

$$
\sigma_{i j}^{(u)}=2 G\left[u_{i j}-\left(u_{k k} / 3\right) \delta_{i j}\right]+K u_{k k} \delta_{i j},
$$

where $u_{i j} \equiv \frac{1}{2}\left(\partial_{i} u_{j}+\partial_{j} u_{i}\right)$ is the network's strain tensor, and $G$ and $K$ its shear and compression moduli, which may be frequency-dependent. The second component is a viscous fluid, having a volume fraction field $\phi_{v}(\mathbf{r}, \omega)$, flow velocity field $\mathbf{v}(\mathbf{r}, \omega)$, pressure field $p(\mathbf{r}, \omega)$, and the stress tensor

$$
\sigma_{i j}^{(v)}=-p \delta_{i j}+2 \eta\left[v_{i j}-\left(v_{k k} / 3\right) \delta_{i j}\right]+\zeta v_{k k} \delta_{i j}
$$

where $v_{i j} \equiv \frac{1}{2}\left(\partial_{i} v_{j}+\partial_{j} v_{i}\right)$ is the fluid's strain-rate tensor, and $\eta$ and $\zeta$ are its shear and compression viscosities. The two components are coupled via mutual friction characterized by a coefficient $\Gamma$. The five governing equations 
for the five fields $\left(\mathbf{u}, \mathbf{v}, p, \phi_{u}, \phi_{v}\right)$ are as follows:

$$
\begin{aligned}
-\omega^{2} \rho_{u} \mathbf{u}= & \nabla \cdot \boldsymbol{\sigma}^{(u)}-\Gamma(i \omega \mathbf{u}-\mathbf{v})+\mathbf{f}_{u} \\
= & G \nabla^{2} \mathbf{u}+(K+G / 3) \nabla(\nabla \cdot \mathbf{u}) \\
& -\Gamma(i \omega \mathbf{u}-\mathbf{v})+\mathbf{f}_{u} \\
i \omega \rho_{v} \mathbf{v}= & \nabla \cdot \boldsymbol{\sigma}^{(v)}-\Gamma(\mathbf{v}-i \omega \mathbf{u})+\mathbf{f}_{v} \\
= & -\nabla p+\eta \nabla^{2} \mathbf{v}+(\zeta+\eta / 3) \nabla(\nabla \cdot \mathbf{v}) \\
& -\Gamma(\mathbf{v}-i \omega \mathbf{u})+\mathbf{f}_{v} \\
0= & \phi_{u}+\nabla \cdot\left(\phi_{u} \mathbf{u}\right) \\
0= & i \omega \phi_{v}+\nabla \cdot\left(\phi_{v} \mathbf{v}\right) \\
1= & \phi_{u}+\phi_{v},
\end{aligned}
$$

where $\rho_{u}, \rho_{v}$ are the mass densities of the two components, and $\mathbf{f}_{u}(\mathbf{r}, \omega), \mathbf{f}_{v}(\mathbf{r}, \omega)$ are external force densities exerted on them. The first two equations, together, reflect the conservation of momentum in the composite material; the third and fourth - the conservation of mass of each component separately; and the last - the assumption of incompressibility for the composite.

We employ the following simplifications. (i) Inertial effects are omitted; they are negligible at sufficiently low frequencies and can easily be included if needed [18. (ii) The network is taken as semidilute, $\phi_{u} \ll \phi_{v} \simeq 1$. (iii) We specialize to the case of no external forces, $\mathbf{f}_{u}=\mathbf{f}_{v}=0$. Under these assumptions the governing equations attain the much simpler form,

$$
\begin{aligned}
& 0=G \nabla^{2} \mathbf{u}+(K+G / 3) \nabla(\nabla \cdot \mathbf{u})-\Gamma(i \omega \mathbf{u}-\mathbf{v}) \\
& 0=-\nabla p+\eta \nabla^{2} \mathbf{v}-\Gamma(\mathbf{v}-i \omega \mathbf{u}) \\
& 0=\nabla \cdot \mathbf{v}
\end{aligned}
$$

The perturbation that we introduce is in the form of a rigid sphere, centered at the origin and moving in the $z$ direction with velocity $\mathbf{U}=U(\omega) \hat{\mathbf{z}}$. Under these conditions the problem has azimuthal symmetry. Hence, using spherical coordinates $(r, \theta, \phi)$, all the fields depend on $r$ and $\theta$ alone, and the vector fields have only $r$ and $\theta$ components. Consequently, the stresses of eqs. (1) and (2) take the form,

$$
\begin{aligned}
\sigma_{r r}^{(u)}= & K\left[\frac{1}{r^{2}} \partial_{r}\left(r^{2} u_{r}\right)+\frac{1}{r \sin \theta} \partial_{\theta}\left(u_{\theta} \sin \theta\right)\right] \\
& +\frac{2 G}{3}\left[2 r \partial_{r}\left(u_{r} / r\right)-\frac{1}{r \sin \theta} \partial_{\theta}\left(u_{\theta} \sin \theta\right)\right] \\
\sigma_{r \theta}^{(u)}= & G\left[r \partial_{r}\left(u_{\theta} / r\right)+\frac{1}{r} \partial_{\theta} u_{r}\right] \\
\sigma_{r r}^{(v)}= & -p+2 \eta \partial_{r} v_{r} \\
\sigma_{r \theta}^{(v)}= & \eta\left[r \partial_{r}\left(v_{\theta} / r\right)+\frac{1}{r} \partial_{\theta} v_{r}\right] .
\end{aligned}
$$

We consider an unbounded medium, which is unperturbed and stationary far away from the sphere. The boundary conditions at infinity are, therefore,

$$
\mathbf{u}(r \rightarrow \infty, \theta)=\mathbf{v}(r \rightarrow \infty, \theta)=\nabla p(r \rightarrow \infty, \theta)=0 .
$$

As to the boundary conditions at the surface of the sphere, we will examine various possibilities in sect. 4 .

\section{General solution}

\subsection{Decoupled flows}

First, we take the divergence of eq. (9) and use eq. (10) to obtain a relation between the compressive stresses of the two components,

$$
\nabla \cdot \mathbf{u}=\frac{1}{i \omega \Gamma} \nabla^{2} p
$$

The next step is to decouple the equations for the two vector fields, $\mathbf{u}$ and $\mathbf{v}$. We apply the following linear transformation:

$$
\begin{aligned}
\mathbf{V}^{(\mathrm{c})} & =\left(1-\frac{\eta}{\eta_{\mathrm{b}}}\right)\left(i \omega \mathbf{u}+\frac{\eta}{\eta_{\mathrm{b}}-\eta} \mathbf{v}-\frac{1}{\Gamma} \nabla p\right) \\
\mathbf{V}^{(\mathrm{r})} & =\left(\frac{\eta_{\mathrm{b}}}{\eta}-1\right)\left(i \omega \mathbf{u}-\mathbf{v}-\frac{1}{\Gamma} \nabla p\right) \\
P & =p-\lambda^{2} \nabla^{2} p
\end{aligned}
$$

whereupon eqs. (8)-(10) turn into

$$
\begin{aligned}
& 0=-\nabla P+\eta_{\mathrm{b}} \nabla^{2} \mathbf{V}^{(\mathrm{c})} \\
& 0=-\nabla P+\eta\left(\nabla^{2} \mathbf{V}^{(\mathrm{r})}-\xi^{-2} \mathbf{V}^{(\mathrm{r})}\right) \\
& 0=\nabla \cdot \mathbf{V}^{(\mathrm{c})}=\nabla \cdot \mathbf{V}^{(\mathrm{r})} .
\end{aligned}
$$

In these equations we have introduced the following bulk viscosity and characteristic lengths:

$$
\begin{aligned}
\eta_{\mathrm{b}} & \equiv G /(i \omega)+\eta \\
\xi & \equiv\left(\frac{G \eta}{i \omega \Gamma \eta_{\mathrm{b}}}\right)^{1 / 2} \\
\lambda & \equiv\left(\frac{K+4 G / 3}{i \omega \Gamma}\right)^{1 / 2}=\left[\frac{2(1-\nu)}{1-2 \nu} \frac{\eta_{\mathrm{b}}}{\eta}\right]^{1 / 2} \xi,
\end{aligned}
$$

where $\nu$ is the network's Poisson ratio.

Thus, the dynamics of the two-fluid model [eqs. (8)(10)] have been decomposed into two independent incompressible flows, $\mathbf{V}^{(\mathrm{c})}$, and $\mathbf{V}^{(\mathrm{r})}$. The former, in which $\mathbf{v}$ and $i \omega \mathbf{u}$ appear with the same sign, describes the collective flow of the network-fluid composite. This flow satisfies the scale-free Stokes equation (17) with viscosity given by the bulk viscosity $\eta_{\mathrm{b}}(\omega)$. The latter, containing $\mathbf{v}$ and $i \omega \mathbf{u}$ with opposite signs, corresponds to the relative flow of the two components. It obeys the Brinkman equation for a fluid embedded in a porous medium [20, eq. (18), depending on the fluid viscosity $\eta$ and characteristic pore size $\xi$. Hence, $\xi$ is identified with the network's mesh size [10, up to a proportionality factor close to unity [12, 13. It also characterizes the spatial decay of transverse (shear) stresses due to the friction between the two components and, therefore, decreases with increasing $\Gamma$. The second length, $\lambda$, characterizes the decay of longitudinal (compressive) stresses and diverges in the limit of an incompressible network $(K \rightarrow \infty$ or $\nu=1 / 2)$. In addition, we define another dynamic length,

$$
\ell_{\mathrm{c}} \equiv\left(\frac{\eta_{\mathrm{b}}}{\eta}\right)^{1 / 2} \xi
$$


As we shall see, $\ell_{c}$ plays a crucial role in the dynamics of the medium. It is proportional to $\lambda$ but remains finite in the limit of an incompressible network. We note the hierarchy $\xi<\ell_{\mathrm{c}}<\lambda$. Usually (e.g., for sufficiently low frequencies $), \eta_{\mathrm{b}} \simeq G /(i \omega) \gg \eta$, and then $\xi \ll \ell_{\mathrm{c}}<\lambda$. Additionally, for a network close to the incompressible limit, we have the complete separation of scales $\xi \ll \ell_{\mathrm{c}} \ll \lambda$.

\subsection{Stream functions and resulting solution}

Following Stokes' original scheme, we express the vector flows in terms of scalar stream functions,

$$
\mathbf{V}^{(\mathrm{c}, \mathrm{r})}=-\frac{1}{r^{2} \sin \theta} \partial_{\theta} \psi^{(\mathrm{c}, \mathrm{r})} \hat{\mathbf{r}}+\frac{1}{r \sin \theta} \partial_{r} \psi^{(\mathrm{c}, \mathrm{r})} \hat{\boldsymbol{\theta}},
$$

and assume the following separation of variables:

$$
\begin{aligned}
\psi^{(\mathrm{c}, \mathrm{r})}(r, \theta) & =f^{(\mathrm{c}, \mathrm{r})}(r) \sin ^{2} \theta \\
p(r, \theta) & =f^{(p)}(r) \cos \theta .
\end{aligned}
$$

Substituting eqs. (24) and (25) in eq. (17) (after taking the curl of the latter to eliminate $\nabla P$ ), we get the equation for $f^{(\mathrm{c})}$,

$$
0=r^{4}\left(f^{(\mathrm{c})}\right)^{\prime \prime \prime \prime}-4 r^{2}\left(f^{(\mathrm{c})}\right)^{\prime \prime}+8 r\left(f^{(\mathrm{c})}\right)^{\prime}-8 f^{(\mathrm{c})} .
$$

Its solution is

$$
f^{(\mathrm{c})}(r)=C_{1}^{(\mathrm{c})} r+\frac{C_{2}^{(\mathrm{c})}}{r}
$$

where we have omitted terms which do not decay to zero after division by $r^{2}$, to ensure a vanishing $\mathbf{V}^{(\mathrm{c})}$ at infinity. The resulting collective flow is

$\mathbf{V}^{(\mathrm{c})}=-2\left(\frac{C_{1}^{(\mathrm{c})}}{r}+\frac{C_{2}^{(\mathrm{c})}}{r^{3}}\right) \cos \theta \hat{\mathbf{r}}+\left(\frac{C_{1}^{(\mathrm{c})}}{r}-\frac{C_{2}^{(\mathrm{c})}}{r^{3}}\right) \sin \theta \hat{\boldsymbol{\theta}}$.

The collective flow contains the $1 / r$ and $1 / r^{3}$ terms known from azimuthally symmetric solutions of the Stokes equation, the first arising from the transverse component of a momentum monopole, and the second from a combination of a momentum quadrupole and a mass dipole 12 .

Repeating the same procedure for the relative flow, we get, instead of eqs. (27)-(29),

$$
\begin{aligned}
0= & r^{4} \xi^{2}\left(f^{(\mathrm{r})}\right)^{\prime \prime \prime \prime}-r^{2}\left(r^{2}+4 \xi^{2}\right)\left(f^{(\mathrm{r})}\right)^{\prime \prime} \\
& +8 r \xi^{2}\left(f^{(\mathrm{r})}\right)^{\prime}+2\left(r^{2}-4 \xi^{2}\right) f^{(\mathrm{r})} \\
f^{(\mathrm{r})}(r)= & \frac{C_{1}^{(\mathrm{r})}}{r}+C_{2}^{(\mathrm{r})}(1+\xi / r) e^{-r / \xi} \\
\mathbf{V}^{(\mathrm{r})}= & -2\left(\frac{C_{1}^{(\mathrm{r})}}{r^{3}}+\frac{C_{2}^{(\mathrm{r})}(r+\xi)}{r^{3}} e^{-r / \xi}\right) \cos \theta \hat{\mathbf{r}} \\
& -\left(\frac{C_{1}^{(\mathrm{r})}}{r^{3}}+\frac{C_{2}^{(\mathrm{r})}\left(r^{2}+\xi r+\xi^{2}\right)}{\xi r^{3}} e^{-r / \xi}\right) \sin \theta \hat{\boldsymbol{\theta}} .
\end{aligned}
$$

The relative flow contains the $1 / r^{3}$ and exponentially small terms known from solutions of the Brinkman equation, the former arising from a mass dipole, and the latter from the spatially decaying transverse momentum, whose decay length is $\xi[21,22,23$.

We substitute eqs. (16), (26), and (29) in eq. (17) to find the equation for $f^{(p)}$,

$0=\lambda^{2} r^{2}\left(f^{(p)}\right)^{\prime \prime}+2 \lambda^{2} r\left(f^{(p)}\right)^{\prime}-\left(r^{2}+2 \lambda^{2}\right) f^{(p)}-2 \eta_{\mathrm{b}} C_{1}^{(\mathrm{c})}$,

whose solution is

$$
f^{(p)}(r)=-\frac{2 \eta_{\mathrm{b}} C_{1}^{(\mathrm{c})}}{r^{2}}+\frac{C^{(p)}(r+\lambda)}{r^{2}} e^{-r / \lambda},
$$

where we have omitted a term that diverges at infinity. The resulting pressure is

$$
p=\left(-\frac{2 \eta_{\mathrm{b}} C_{1}^{(\mathrm{c})}}{r^{2}}+\frac{C^{(p)}(r+\lambda)}{r^{2}} e^{-r / \lambda}\right) \cos \theta .
$$

It contains a $1 / r^{2}$ term, arising from the longitudinal component of a momentum monopole, and exponentially small terms, corresponding to spatially decaying longitudinal stresses whose decay length is $\lambda$. The transformed pressure $P$ [eq. (16)] contains only the $1 / r^{2}$ term.

We can use eq. (18) for the relative flow, rather than eq. (17) for the collective one, and obtain another expression for $f^{(p)}$. Equating the two expressions gives a relation between $C_{1}^{(\mathrm{r})}$ and $C_{1}^{(\mathrm{c})}$,

$$
C_{1}^{(\mathrm{r})}=\left(2 \eta_{\mathrm{b}} \xi^{2} / \eta\right) C_{1}^{(\mathrm{c})}=2 \ell_{\mathrm{c}}^{2} C_{1}^{(\mathrm{c})} .
$$

This is the first explicit appearance of the dynamic length $\ell_{\mathrm{c}}$, relating the leading terms in the collective and relative flows.

The general solution that is regular at infinity contains four integration constants $\left(C_{1}^{(\mathrm{c})}, C_{2}^{(\mathrm{c})}, C_{2}^{(\mathrm{r})}, C^{(p)}\right)$ to be determined in sect. 4 from boundary conditions. However, we first present several quantities of interest in terms of the undetermined constants. We use them to derive properties stemming from the fundamental response of the medium - in fact, any isotropic viscoelastic medium independent of specific boundary conditions.

\subsection{Properties of the general solution}

The general solution for $\mathbf{V}^{(\mathrm{c}, \mathrm{r})}$, eqs. (29) and (32), can be substituted back in eqs. (14) and (15) to obtain the original fields, $\mathbf{u}(r, \theta)$ and $\mathbf{v}(r, \theta)$. The resulting expressions can be found in the Supplementary Material 24.

The first quantity of interest is the total force $\mathbf{F}$ exerted on the sphere. We substitute the general solution for $\mathbf{u}$ and $\mathbf{v}$ in the stress tensors, eq. (11), and integrate the stresses over the surface of the sphere to obtain the force. The separate network and fluid contributions to the force are found in ref. 24. Once they are added together, most terms vanish, leaving

$$
\mathbf{F}=-8 \pi \eta_{\mathrm{b}} C_{1}^{(\mathrm{c})} \hat{\mathbf{z}} .
$$


Recall that $C_{1}^{(\mathrm{c})}$ is associated with the long-range $1 / r$ term in the collective flow, eq. (29), and the corresponding longrange $1 / r^{2}$ term in the pressure, eq. (35). The simple form of eq. (37) stems from momentum conservation - at large distances, only the $1 / r$ collective flow is at play, and it corresponds to the flow due to a momentum monopole $\mathbf{F}$, with effective viscosity $\eta_{\mathrm{b}}$.

Let us examine the large-distance behavior of the flows in more detail. The asymptotic term in eq. (29), as well as those extracted from the expressions for $\mathbf{u}$ and $\mathbf{v}$ are

$$
\mathbf{V}^{(\mathrm{c}, 0)}=\mathbf{v}^{(0)}=i \omega \mathbf{u}^{(0)}=-\frac{C_{1}^{(\mathrm{c})}}{r}(2 \cos \theta \hat{\mathbf{r}}-\sin \theta \hat{\boldsymbol{\theta}}),
$$

whereas $\mathbf{V}^{(\mathrm{r}, 0)}=0$. Thus, far away from the perturbation the two components flow together. Combining eqs. (37) and (38), we find that, in terms of the force $\mathbf{F}$, the large-distance flow has a universal form independent of boundary conditions, mesh size, and compressibility,

$$
\mathbf{V}^{(\mathrm{c}, 0)}=\mathbf{v}^{(0)}=i \omega \mathbf{u}^{(0)}=\frac{1}{8 \pi \eta_{\mathrm{b}} r}\left(2 F_{r} \hat{\mathbf{r}}+F_{\theta} \hat{\boldsymbol{\theta}}\right)
$$

It represents a generalized Oseen tensor, with $\eta_{\mathrm{b}}$ replacing $\eta$, which is the basis of the original two-point microrheology [3. Its universality stems, once again, from the conservation at large distance of transverse momentum emanating from the source $\mathbf{F}$. The Oseen tensor describes a longitudinal flow response (relation between the $r$-components of the force and velocity) and a transverse response (relation between the $\theta$-components), which are both positive. That is, if a second particle is placed along or perpendicular to the direction of the sphere's motion, in both cases it will be advected in the same direction. The general result expressed in eq. (39) disagrees with earlier theoretical ones concerning the far-field flow [9,10,11]. In refs. [10,11] the far field was found to depend on network compressibility. (Indeed, it was suggested as a means to extract the compressibility from two-point correlations.) In ref. 9] it was shown to depend on network compressibility, as well as the specific choice of boundary conditions, thus undermining the universality of asymptotic two-point microrheology. The apparent contradictions between these results and ours are discussed, and resolved, in sect. 5.

We are interested also in the sub-asymptotic $1 / r^{3}$ terms,

$$
\begin{aligned}
& \mathbf{V}^{(\mathrm{c}, 1)}=-\frac{C_{2}^{(\mathrm{c})}}{r^{3}}(2 \cos \theta \hat{\mathbf{r}}+\sin \theta \hat{\boldsymbol{\theta}}) \\
& \mathbf{V}^{(\mathrm{r}, 1)}=-\frac{2 \ell_{\mathrm{c}}^{2} C_{1}^{(\mathrm{c})}}{r^{3}}(2 \cos \theta \hat{\mathbf{r}}+\sin \theta \hat{\boldsymbol{\theta}}),
\end{aligned}
$$

where we have used eq. (36) to replace $C_{1}^{(\mathrm{r})}$ with $C_{1}^{(\mathrm{c})}$. The sub-asymptotic terms describe also relative motion of the two components. The terms in the collective and relative flows depend on different coefficients. In the case of the relative flow, we can use eq. (37) to substitute $C_{1}^{(\mathrm{c})}$ in eq. (40) and obtain a universal form in terms of $\mathbf{F}$,

$$
\mathbf{V}^{(\mathrm{r}, 1)}=\frac{\ell_{\mathrm{c}}^{2}}{4 \pi \eta_{\mathrm{b}} r^{3}}\left(2 F_{r} \hat{\mathbf{r}}-F_{\theta} \hat{\boldsymbol{\theta}}\right) .
$$

This expression describes the flow created by a mass dipole equal to $-\left(\ell_{\mathrm{c}}^{2} / \eta_{\mathrm{b}}\right) \mathbf{F}=-\left(\xi^{2} / \eta\right) \mathbf{F}$. It is the large-distance flow caused by a point force in a Brinkman fluid [21,22, 23 . One of its footprints is its sign, implying a positive effect on the longitudinal response and a negative effect on the transverse one. Comparing eqs. (39) and (41), we identify $\sqrt{2} \ell_{\mathrm{c}}$ as the distance at which the leading terms in the collective and relative flows become equal.

The original flow fields, which are more accessible experimentally, contain combinations of sub-asymptotic terms from both the collective and relative flows,

$$
\begin{aligned}
\mathbf{v}^{(1)} & =-\frac{C_{2}^{(\mathrm{c})}-2 \xi^{2} C_{1}^{(\mathrm{c})}}{r^{3}}(2 \cos \theta \hat{\mathbf{r}}+\sin \theta \hat{\boldsymbol{\theta}}) \\
i \omega \mathbf{u}^{(1)} & =-\frac{C_{2}^{(\mathrm{c})}-2\left(\ell_{\mathrm{c}}^{2}+\xi^{2}\right) C_{1}^{(\mathrm{c})}}{r^{3}}(2 \cos \theta \hat{\mathbf{r}}+\sin \theta \hat{\boldsymbol{\theta}}),
\end{aligned}
$$

such that $\mathbf{v}^{(1)}-i \omega \mathbf{u}^{(1)}=\mathbf{V}^{(\mathrm{r}, 1)}$, the universal expression of eq. (41). Because of these combinations, the observed distances of crossover between the sub-asymptotic and asymptotic behaviors do not necessarily coincide with $\ell_{\mathrm{c}}$. Hence, we define two such distances, $r_{\mathrm{c}}^{(v)}$ and $r_{\mathrm{c}}^{(u)}$, as those at which $\left|\mathbf{v}^{(0)}\right|=\left|\mathbf{v}^{(1)}\right|$ and $\left|\mathbf{u}^{(0)}\right|=\left|\mathbf{u}^{(1)}\right|$, respectively. According to eqs. (38) and (42) this occurs for

$$
r_{\mathrm{c}}^{(v)}=\left[\left(r_{\mathrm{c}}^{(u)}\right)^{2}+2 \ell_{\mathrm{c}}^{2}\right]^{1 / 2}=\left|C_{2}^{(\mathrm{c})} / C_{1}^{(\mathrm{c})}-2 \xi^{2}\right|^{1 / 2} .
$$

\section{Particular solutions for different boundary conditions}

The general solution obtained in the preceding section, after imposing the boundary conditions at infinity, contains four integration constants, $\left(C_{1}^{(\mathrm{c})}, C_{2}^{(\mathrm{c})}, C_{2}^{(\mathrm{r})}, C^{(p)}\right)$. Four boundary conditions are required, therefore, at the sphere surface. In the following we consider three cases for these boundary conditions. They correspond to different physical conditions for the interaction between the sphere and the network, as will be discussed in sect. 5. Other cases can be readily studied using the file provided as Supplementary Material [24].

\subsection{Sticking fluid and network}

The first case that we study is stick boundary conditions for both network and fluid, i.e., the velocities of the two components at the sphere surface are equal to the velocity of the sphere,

$$
\mathbf{v}(a, \theta)=i \omega \mathbf{u}(a, \theta)=U \hat{\mathbf{z}} .
$$

We substitute these four conditions in the expressions for $\mathbf{u}$ and $\mathbf{v}$, and solve for the four constants [24].

Using eq. (37), we find for the total friction coefficient, $\gamma \equiv F / U$

$$
\frac{\gamma}{6 \pi \eta_{\mathrm{b}} a}=1-\frac{\ell_{\mathrm{c}}^{2}-\xi^{2}}{a^{2}+2 \lambda^{2}+2 \lambda a+\xi a+\ell_{\mathrm{c}}^{2}} .
$$


Recalling the hierarchy $\xi<\ell_{\mathrm{c}}<\lambda$, we find that deviations from the GSR can only be negative, and we also identify two relevant limits. For a very large sphere,

$$
a \gg \ell_{\mathrm{c}}: \quad \gamma \simeq 6 \pi \eta_{\mathrm{b}} a,
$$

$\gamma$ obeys, as expected, the generalized Stokes relation (GSR) with viscosity $\eta_{\mathrm{b}}(\omega)$. The condition for eq. (46) to hold, however, is harder to fulfill than what is usually assumed, since $\ell_{c}$ may be much larger than the mesh size $\xi$. For smaller spheres,

$$
a \ll \ell_{\mathrm{c}}: \quad \frac{\gamma}{6 \pi \eta_{\mathrm{b}} a} \simeq 1-\frac{\ell_{\mathrm{c}}^{2}}{2 \lambda^{2}+\ell_{\mathrm{c}}^{2}}=\frac{4(1-\nu)}{5-6 \nu},
$$

where we have used eqs. (22) and (23). Equation (47) describes the decrease of $\gamma$ due to network compressibility. (See fig. 2(a).) The reduction is not sharp; for a Poisson ratio $\nu=0.4$ it amounts to less than $10 \%$. In the limit of an incompressible network, for these boundary conditions, the GSR holds for any value of $a$.

Note that the transition from eq. (46) to eq. (47) upon decreasing $a$ can be recast as a transition with decreasing frequency; as $\omega$ gets smaller, the ratio $\eta_{\mathrm{b}}(\omega) / \eta$ increases, making eq. (47) with its compressibility correction the valid one. This is in line with the picture presented in ref. 9 .

The asymptotic flow has its universal form, eq. (39). The full expressions for the sub-asymptotic flow and the resulting crossover lengths are given in ref. 24]. Assuming that $\xi$ is the smallest length, there are two limiting cases. For a large sphere,

$$
\begin{array}{cl}
a \gg \ell_{\mathrm{c}}: & i \omega \mathbf{u}^{(1)} \simeq \mathbf{v}^{(1)} \simeq-\frac{a^{2}}{24 \pi \eta_{\mathrm{b}} r^{3}}\left(2 F_{r} \hat{\mathbf{r}}-F_{\theta} \hat{\boldsymbol{\theta}}\right) \\
& r_{\mathrm{c}}^{(v)} \simeq r_{\mathrm{c}}^{(u)} \simeq a / \sqrt{3} .
\end{array}
$$

Thus, in this limit, also the sub-asymptotic terms describe collective flow of the two components together. [This is only approximate, though, since there is always the universal relative flow of eq. (41).] These terms set in only in the near field and, therefore, are not of much interest. Equations (39) and (48) are identical to the Stokes flow due to a sphere moving in a fluid of viscosity $\eta_{\mathrm{b}}$. For a smaller sphere,

$$
\begin{aligned}
a \ll \ell_{\mathrm{c}}: & \mathbf{v}^{(1)} \simeq-\frac{a^{2}}{48(1-\nu) \pi \eta_{\mathrm{b}} r^{3}}\left(2 F_{r} \hat{\mathbf{r}}-F_{\theta} \hat{\boldsymbol{\theta}}\right) \\
& i \omega \mathbf{u}^{(1)} \simeq-\frac{\ell_{\mathrm{c}}^{2}}{4 \pi \eta_{\mathrm{b}} r^{3}}\left(2 F_{r} \hat{\mathbf{r}}-F_{\theta} \hat{\boldsymbol{\theta}}\right) \\
& r_{\mathrm{c}}^{(v)} \simeq a / \sqrt{6(1-\nu)} \\
& r_{\mathrm{c}}^{(u)} \simeq \sqrt{2} \ell_{\mathrm{c}} .
\end{aligned}
$$

In this limit the two components flow with different velocities. The network flow is larger and crosses over to the asymptotic one at a larger distance. The sign of the sub-asymptotic terms in eqs. (48) and (49) indicates the dominance of the force-quadrupole contribution to these terms [12, leading to a negative correction to the longitudinal response and a positive correction to the transverse one.

\subsection{Sticking fluid and slipping network}

If the network is allowed to fully slip over the sphere surface, the four boundary conditions become

$$
\mathbf{v}(a, \theta)=U \hat{\mathbf{z}}, \quad i \omega u_{r}(a, \theta)=U \cos \theta, \quad \sigma_{r \theta}^{(u)}(a, \theta)=0 .
$$

The friction coefficient in this case turns out as

$$
\begin{aligned}
& \frac{\gamma}{6 \pi \eta_{\mathrm{b}} a}= \\
& 1-\frac{\left(\ell_{\mathrm{c}}^{2}-\xi^{2}\right)\left(a^{2}+2 \lambda^{2}+2 a \lambda+2 \ell_{\mathrm{c}}^{2}\right)}{\left(a^{2}+2 \lambda^{2}+2 \lambda a\right)\left(a \xi+3 \ell_{\mathrm{c}}^{2}\right)+2 \ell_{\mathrm{c}}^{2}\left(a \xi+\ell_{\mathrm{c}}^{2}\right)} .
\end{aligned}
$$

Analysis of eq. (51) leads to the following three limits. For a sufficiently large sphere, satisfying either $a \gg \max \left(\ell_{\mathrm{c}}^{2} / \xi, \lambda\right)$ or $\ell_{\mathrm{c}}^{2} / \xi \ll a \ll \lambda$, we obtain the GSR of eq. (46). This condition is even harder to fulfill than the one in sect. 4.1. For a smaller sphere, we obtain a modified relation,

$$
a \ll \min \left(\ell_{\mathrm{c}}^{2} / \xi, \lambda\right): \frac{\gamma}{6 \pi \eta_{\mathrm{b}} a} \simeq \frac{2 \lambda^{2}}{3 \lambda^{2}+\ell_{\mathrm{c}}^{2}}=\frac{4(1-\nu)}{7-8 \nu} .
$$

In the limit of an incompressible network this expression describes a sphere with slip boundary conditions in a fluid of viscosity $\eta_{\mathrm{b}}(\omega), \gamma=4 \pi \eta_{\mathrm{b}} a$. The third limit, $\lambda \ll a \ll$ $\ell_{\mathrm{c}}^{2} / \xi$, gives the same compressibility-independent full-slip result. Note again that the same change from the GSR [eq. (46)] to a slip- and compressibility-dependent relation [eq. (52)] is achieved also by decreasing $\omega$, as noted in ref. [9].

The asymptotic flow remains that of eq. (39). We give the full expressions for the sub-asymptotic flow and crossover lengths in ref. 24]. Assuming again that $\xi$ is the smallest length, there are two limiting cases. For a very large sphere,

$$
\begin{aligned}
a \gg \lambda: \quad \mathbf{v}^{(1)} & \simeq-\frac{1}{2} i \omega \mathbf{u}^{(1)} \simeq \frac{\ell_{\mathrm{c}}^{2}}{12 \pi \eta_{\mathrm{b}} r^{3}}\left(2 F_{r} \hat{\mathbf{r}}-F_{\theta} \hat{\boldsymbol{\theta}}\right) \\
r_{\mathrm{c}}^{(v)} & \simeq r_{\mathrm{c}}^{(u)} / \sqrt{2} \simeq \sqrt{2 / 3} \ell_{\mathrm{c}} .
\end{aligned}
$$

For a smaller sphere,

$$
\begin{aligned}
& a \ll \lambda: \quad \mathbf{v}^{(1)} \simeq \frac{a^{2}(1-2 \nu)}{48(1-\nu) \pi \eta_{\mathrm{b}} r^{3}}\left(2 F_{r} \hat{\mathbf{r}}-F_{\theta} \hat{\boldsymbol{\theta}}\right) \\
& i \omega \mathbf{u}^{(1)} \simeq-\frac{\ell_{\mathrm{c}}^{2}}{4 \pi \eta_{\mathrm{b}} r^{3}}\left(2 F_{r} \hat{\mathbf{r}}-F_{\theta} \hat{\boldsymbol{\theta}}\right) \\
& r_{\mathrm{c}}^{(v)} \simeq \sqrt{(1-2 \nu) /[6(1-\nu)]} a \\
& r_{\mathrm{c}}^{(u)} \simeq \sqrt{2} \ell_{\mathrm{c}} .
\end{aligned}
$$

Therefore, for the case of a slipping network we find in the sub-asymptotic region relative flow of the two components. Note the change of sign of the sub-asymptotic $\mathbf{v}^{(1)}$ in eqs. (53) and (54) as compared to sect. 4.1. This marks the dominance of the mass-dipole effect in these $1 / r^{3}$ terms, leading to a positive correction to the longitudinal response and a negative correction to the transverse one. Yet, as in sect. 4.1 these effects are of limited relevance as they set in at distances comparable to the particle size. 


\subsection{Sticking fluid and free network}

The third and last case that we consider is a free network, i.e., one that does not directly exchange stresses with the sphere. The network moves only because of its coupling to the fluid. Discussing the physical relevance of these conditions is deferred to sect. 5. The corresponding four boundary conditions are

$$
\mathbf{v}(a, \theta)=U \hat{\mathbf{z}}, \quad \sigma_{r r}^{(u)}(a, \theta)=\sigma_{r \theta}^{(u)}(a, \theta)=0 .
$$

Here, the force exerted on the sphere comes solely from the fluid component, without contribution from the network. The friction coefficient turns out as

$$
\begin{aligned}
\frac{\gamma}{6 \pi \eta_{\mathrm{b}} a} & =\frac{a^{2} \lambda(a+\lambda)\left(a \xi+\xi^{2}+2 \ell_{\mathrm{c}}^{2}\right)+}{a^{2} \lambda(a+\lambda)\left(a \xi+3 \ell_{\mathrm{c}}^{2}\right)+} \cdots \\
& \ldots \frac{+2 \ell_{\mathrm{c}}^{2} \xi(a+3 \lambda)(2 a+3 \lambda)(a+\xi)}{+2 \ell_{\mathrm{c}}^{2}(a+3 \lambda)(2 a+3 \lambda)\left(a \xi+\ell_{\mathrm{c}}^{2}\right)} .
\end{aligned}
$$

The condition of a free network should be appropriate, in particular, for beads smaller than the mesh size. Hence, unlike the preceding two sub-sections, we do not assume that $\xi$ is the smallest scale. This leads to three limiting cases. For a very large sphere, satisfying either $a \gg$ $\max \left(\ell_{\mathrm{c}}^{2} / \xi, \lambda\right)$ or $\ell_{\mathrm{c}}^{2} / \xi \ll a \ll \lambda$, the GSR of eq. (46) is recovered. For smaller spheres, where $\ell_{\mathrm{c}} \ll a \ll \min \left(\ell_{\mathrm{c}}^{2} / \xi, \lambda\right)$ or $\lambda \ll a \ll \ell_{\mathrm{c}}^{2} / \xi$, the effective-slip result is obtained, $\gamma \simeq 4 \pi \eta_{\mathrm{b}} a$. For yet smaller spheres, we get

$$
a \ll \ell_{\mathrm{c}}: \quad \gamma \simeq 6 \pi \eta a\left[a^{2} /\left(9 \xi^{2}\right)+a / \xi+1\right] .
$$

For the current boundary conditions, and spheres smaller than the mesh size, eq. (57) enables us to recover the ordinary Stokes relation, $\gamma \simeq 6 \pi \eta a$. For spheres of intermediate size, $\xi \ll a \ll \ell_{\mathrm{c}}$, a different relation is obtained from eq. (57), $\gamma \simeq(2 / 3) \pi \eta a^{3} / \xi^{2}$. Note that both of these limiting expressions are independent of compressibility.

The asymptotic flow remains in its universal form, eq. (39). The full expressions for the sub-asymptotic flows and crossover distances are found in ref. 24. We present results in the following two opposite limits. For very large spheres the sub-asymptotic flows and crossover distances coincide with those of a sphere in an ordinary Stokes flow as found in sect. 4.1, eq. (48). For small spheres, however, we obtain

$$
\begin{aligned}
a \ll \ell_{\mathrm{c}}: \mathbf{v}^{(1)} & \simeq \frac{3 \ell_{\mathrm{c}}^{2}\left[a^{2}+3 \xi(a+\xi)\right]}{4 \pi \eta_{\mathrm{b}}\left[a^{2}+9 \xi(a+\xi)\right] r^{3}}\left(2 F_{r} \hat{\mathbf{r}}-F_{\theta} \hat{\boldsymbol{\theta}}\right) \\
i \omega \mathbf{u}^{(1)} & \simeq \frac{\ell_{\mathrm{c}}^{2} a^{2}}{2 \pi \eta_{\mathrm{b}}\left[a^{2}+9 \xi(a+\xi)\right] r^{3}}\left(2 F_{r} \hat{\mathbf{r}}-F_{\theta} \hat{\boldsymbol{\theta}}\right) \\
r_{\mathrm{c}}^{(v)} & \simeq\left(\frac{6\left[a^{2}+3 \xi(a+\xi)\right]}{a^{2}+9 \xi(a+\xi)}\right)^{1 / 2} \ell_{\mathrm{c}} \\
r_{\mathrm{c}}^{(u)} & \simeq \frac{2 a}{\left[a^{2}+9 \xi(a+\xi)\right]^{1 / 2}} \ell_{\mathrm{c}} .
\end{aligned}
$$

As in sect. 4.2, the sign of the sub-asymptotic flows for $a \ll \ell_{\mathrm{c}}$ indicates the dominance of the mass-dipole contribution to the $1 / r^{3}$ terms. However, in the present case, the effect is amplified by a much larger mass dipole, proportional to $\ell_{\mathrm{c}}^{2} / \eta_{\mathrm{b}}=\xi^{2} / \eta$. This is because the bead displaces only the local fluid as it oscillates without contact with the network. Consequently, we expect large positive and negative corrections, respectively, to the longitudinal and transverse responses, pushing the crossover between the sub-asymptotic and asymptotic behaviors further away to a distance $\sim \ell_{\mathrm{c}}$, much larger than both $\xi$ and $a$.

\subsection{Scaling relations}

The problem which we have been studying depends on three lengths $-a, \xi, \lambda$ - and two viscosities $-\eta, \eta_{\mathrm{b}}$. (Equivalently, one of the viscosities can be traded for the fourth length $\ell_{c}$.) Any quantity of interest, such as $\gamma, r^{3} v^{(1)}$, or $r_{\mathrm{c}}^{(u, v)}$, once properly scaled, can be expressed as a dimensionless function of $\xi / a, \lambda / a$, and $\eta_{\mathrm{b}} / \eta$. If we assume the limits of large $\lambda / a$ and $\eta_{\mathrm{b}} / \eta$ (both satisfied in most practical circumstances), we are left with functions of $\xi / a \equiv x$ alone. Such single-variable scaling functions are useful for comparison between experiment and theory [12] and for reliable extraction of material parameters, such as the correlation length $\xi$ [13. We concentrate on the properties $\gamma$, $\mathbf{v}^{(1)}$, and $r_{\mathrm{c}}^{(v)}$, in the limit $a \ll \ell_{\mathrm{c}}$, as these are the most relevant experimentally.

We begin with the sphere's friction coefficient $\gamma$, which is directly measured in one-point microrheology. We define the scaling function $\tilde{\gamma}_{i} \equiv \gamma_{i} /\left(6 \pi \eta_{\mathrm{b}} a\right)$, where the index $i=$ $1,2,3$ corresponds to the three sets of boundary conditions treated in sects.4.1, 4.2 and 4.3. From eqs. (47), (52), and (57) we find, respectively,

$$
\begin{aligned}
\tilde{\gamma}_{1}(x) & =4(1-\nu) /(5-6 \nu)=\mathrm{const} \\
\tilde{\gamma}_{2}(x) & =4(1-\nu) /(7-8 \nu)=\mathrm{const} \\
\left(\eta_{\mathrm{b}} / \eta\right) \tilde{\gamma}_{3}(x) & =[1 / 9+x(1+x)] / x^{2} .
\end{aligned}
$$

We turn next to the sub-asymptotic flow $\mathbf{v}^{(1)}$ and focus on its radial component at zero angle, $v_{r}^{(1)}(r, 0)$. This function can be measured from the longitudinal displacement correlations of particle pairs in the extended twopoint technique [12,13]. We define the scaling function $\tilde{v}_{i}^{(1)} \equiv\left[4 \pi \eta_{\mathrm{b}} /\left(a^{2} F_{r}\right)\right] r^{3} v_{r, i}^{(1)}$. Equations (49), (54), and (58) yield, respectively,

$$
\begin{aligned}
\tilde{v}_{1}^{(1)}(x) & =-1 /[6(1-\nu)]=\mathrm{const} \\
\tilde{v}_{2}^{(1)}(x) & =(1-2 \nu) /[6(1-\nu)]=\mathrm{const} \\
\left(\eta / \eta_{\mathrm{b}}\right) \tilde{v}_{3}^{(1)}(x) & =6 x^{2}[1+3 x(1+x)] /[1+9 x(1+x)] .
\end{aligned}
$$

Finally, we address the crossover distance $r_{\mathrm{c}}^{(v)}$. Defining $\tilde{r}_{\mathrm{c}, i}^{(v)} \equiv r_{\mathrm{c}, i}^{(v)} / a$, we find from eqs. (49), (54) and (58) the simple relation

$$
\tilde{r}_{\mathrm{c}, i}^{(v)}(x)=\left|\tilde{v}_{i}^{(1)}(x)\right|^{1 / 2},
$$

where the functions $\tilde{v}_{i}^{(1)}(x)$ are given in eq. (60). 
In the case of the third set of boundary conditions (sticking fluid and free network) the rescaled sub-asymptotic flow and crossover distance, $\tilde{v}_{3}^{(1)}$ and $\tilde{r}_{\mathrm{c}, 3}^{(v)}$, depend on the fluid viscosity $\eta$. As will be discussed in sect. 5 this makes comparison between the two-fluid model and experiment problematic. We would like to replace the solvent's $\eta$ with the effective local viscosity sensed by the sphere, $\eta_{l}, e . g$., as inferred from one-point microrheology using the definition $\eta_{l} \equiv \gamma /(6 \pi a)$. Using eq. (56) we make this replacement and obtain

$$
\left(\tilde{r}_{\mathrm{c}, 3}^{(v)}\right)^{2}=\tilde{v}_{3}^{(1)}=2\left(\eta_{\mathrm{b}} / \eta_{l}\right)\left(x^{2}+x+1 / 3\right) .
$$

\section{Discussion}

\subsection{Network-particle boundary conditions}

We have formulated the particle-medium coupling in terms of boundary conditions at the sphere surface, without abandoning the continuum description. While the solvent has been assumed to satisfy the stick boundary condition, three different sets of boundary conditions have been assumed for the network. On top of the previously studied network stick and slip 9, we have introduced boundary conditions corresponding to a free network that does not exchange stresses directly with the sphere. We expect the different boundary conditions to hold in different experimental scenarios. In cases where the network is bound to the sphere, stick boundary conditions should clearly hold. In scenarios where the sphere is surrounded by solvent without direct contact with the network, the freenetwork boundary conditions should be valid. This will happen, for example, for particles much smaller than the network mesh size and for those moving inside a solvent "cage". It is unclear what boundary conditions should be used in-between these two extremes of strong contact and no contact. For flexible polymer networks, which are neither bound to nor depleted from the bead, the appropriate boundary conditions might be those of full or partial slip [7.

We have focused on the effect of these different particlemedium couplings on the following dynamic properties: (a) the sphere's friction coefficient $\gamma$ (equivalently, its displacement autocorrelation in equilibrium); (b) the asymptotically far flow field $\mathbf{v}^{(0)}$ created by the sphere's motion (equivalently, the displacement pair correlation function at large separations); (c) the sub-asymptotic flow field $\mathbf{v}^{(1)}$ (displacement pair correlation function at intermediate separations). All three properties are directly accessible by microrheology - the first through one-point measurements, and the other two through two-point ones. We now discuss the results for these three properties.

\subsection{Local dynamics}

The results for $\gamma$ underline the sensitivity of this one-point property to the immediate environment of the particle, as was indicated by earlier studies $[3,5,7,9,10,11,18$. We find that the GSR - the assumption underlying one-point microrheology - is generally violated. It is valid only in two quite strict limits: (a) a very large sphere, whose size exceeds $\ell_{\mathrm{c}}$ or $\lambda$, i.e., a length proportional to the mesh size times a large factor dependent on $\eta_{\mathrm{b}}(\omega) / \eta$; or (b) a smaller sphere comparable to the mesh size together with a very high frequency, such that $\eta_{\mathrm{b}}(\omega) \simeq \eta$. Otherwise, there are significant deviations from the GSR, as given by eqs. (47), (52), and (57). Naturally, the deviation is particularly large for the free-network boundary conditions [eq. (57)], where the bulk viscosity $\eta_{\mathrm{b}}(\omega)$ is replaced by a local viscosity which is usually much smaller. (Theoretically, according to eq. (57), it should be the solvent viscosity $\eta$; yet, this is not so in practice. See the discussion below.)

\subsection{Far flow}

The results for $\mathbf{v}^{(0)}$, by contrast, are universal. The asymptotic flow always obeys, exactly, a generalized Oseen tensor for a fluid with effective shear viscosity $\eta_{\mathrm{b}}(\omega)$, eq. (39), which is independent of boundary conditions and network compressibility. This finding is in apparent contradiction with earlier theoretical results, which we now discuss in detail.

Levine and Lubensky observed that the component of the two-point correlations perpendicular to the line connecting the two points, at large separations, depended on compressibility [10,11]. They suggested, therefore, to extract the compressibility from the ratio of perpendicular to parallel components of two-point measurements. The reason for the appearance of compressibility in their asymptotic expressions was that they considered a compressible viscoelastic bulk, whereas in the two-fluid model there is a background of incompressible fluid. As shown above, at large distances the network and its solvent move together; the relevant compressibility, therefore, is that of the medium as a whole (essentially the solvent's), which is, to a very good approximation, zero. The suggestion made in refs. [10,11] was subsequently tried experimentally [6, 25. A Poisson ratio of 0.5 (negligible compressibility) was measured for actin networks [6, 25, and a slightly smaller value for a mixture of actin and microtubules [25]. Thus, a reliable microrheology measurement of the compressibility of biopolymer networks has not been achieved [26]. According to our analysis [eq. (39)] this is simply because the $1 / r$ pair correlations at large distances do not depend at all on network compressibility; the ratio of perpendicular to parallel components of this correlation is invariably $1 / 2$.

According to Fu et al. 9] the far flow depends on the particular choice of boundary conditions at the sphere surface. This disagreement with our results is readily resolved once we notice that the universality of the far flow appears when we prescribe the force $\mathbf{F}$ acting on the sphere, whereas they prescribed the sphere's velocity. In other words, the dependence of the far flow on boundary conditions in ref. [9] arises only from the dependence of $\gamma$ (i.e., the single-particle force-velocity relation) on those 
boundary conditions. We note that the flow velocity as a function of force is the one related to the particles' pair mobility and, hence, the one relevant to two-point measurements.

On the one hand, the universality of $\mathbf{v}^{(0)}$ reinforces the robustness of asymptotic two-point microrheology for measuring bulk shear moduli. On the other hand, the pair correlations at asymptotically large distances cannot reveal separate properties of the network and solvent such as network compressibility. A way to measure the compressibility is outlined below in sect. 5.7

\subsection{Intermediate flow}

The form of the relative flow at intermediate distances is universal as well, assuming that one knows $\ell_{\mathrm{c}}=\left(\eta_{\mathrm{b}} / \eta\right)^{1 / 2} \xi$; cf. eq. (41). However, the sub-asymptotic flow accessible to two-point experiments, $\mathbf{v}^{(1)}$, does depend on boundary conditions. In the case of sticking or slipping network it is of limited interest since it sets in at distances comparable to the particle size. In the free-network case the massdipole effect causing the sub-asymptotic flow is strongly enhanced, as the bulk viscosity is replaced by the local one. This makes the $1 / r^{3}$ corrections to the asymptotic $1 / r$ terms significant far from the sphere, making them manifest in two-point microrheology [12].

\subsection{Biopolymer networks}

The results for the three sets of boundary conditions, therefore, differ considerably. In particular, the free-network case strongly deviates from those of sticking and slipping networks. This is demonstrated in fig.2 for the dependence of the friction coefficient $\gamma$ on network compressibility, and the dependence of the observable crossover distance $r_{\mathrm{c}}^{(v)}$ on the mesh size $\xi$.

In the special case of entangled F-actin networks, the appropriate boundary conditions, even for spheres significantly larger than the mesh size, are those of a free network. The strong evidence for this unintuitive result comes from agreement with experiments [12,13] concerning the large prefactor of $\eta_{\mathrm{b}}(\omega) / \eta$, which enhances the intermediate flow $\mathbf{v}^{(1)}$ and squared crossover length $\left(r_{\mathrm{c}}^{(v)}\right)^{2}$ compared to the other boundary conditions. [See eqs. (58) and (60).] Given the large differences in the results between the free-network conditions and the other two sets (see fig. 2, for example), there is no way that stick or slip boundary conditions could fit the experimental data. The same seems to hold for microtubule networks, where a crossover length much larger than the mesh size was observed as well 25. As mentioned above, the free-network boundary condition leads also to the largest downward deviation of $\gamma$ from the GSR. This accounts for the ordersof-magnitude discrepancies found between one- and twopoint (or macroscopic) measurements in actin $3,5,6,7$ and microtubule [25] networks, as well as the sensitivity of one-point measurements to the surface chemistry of the
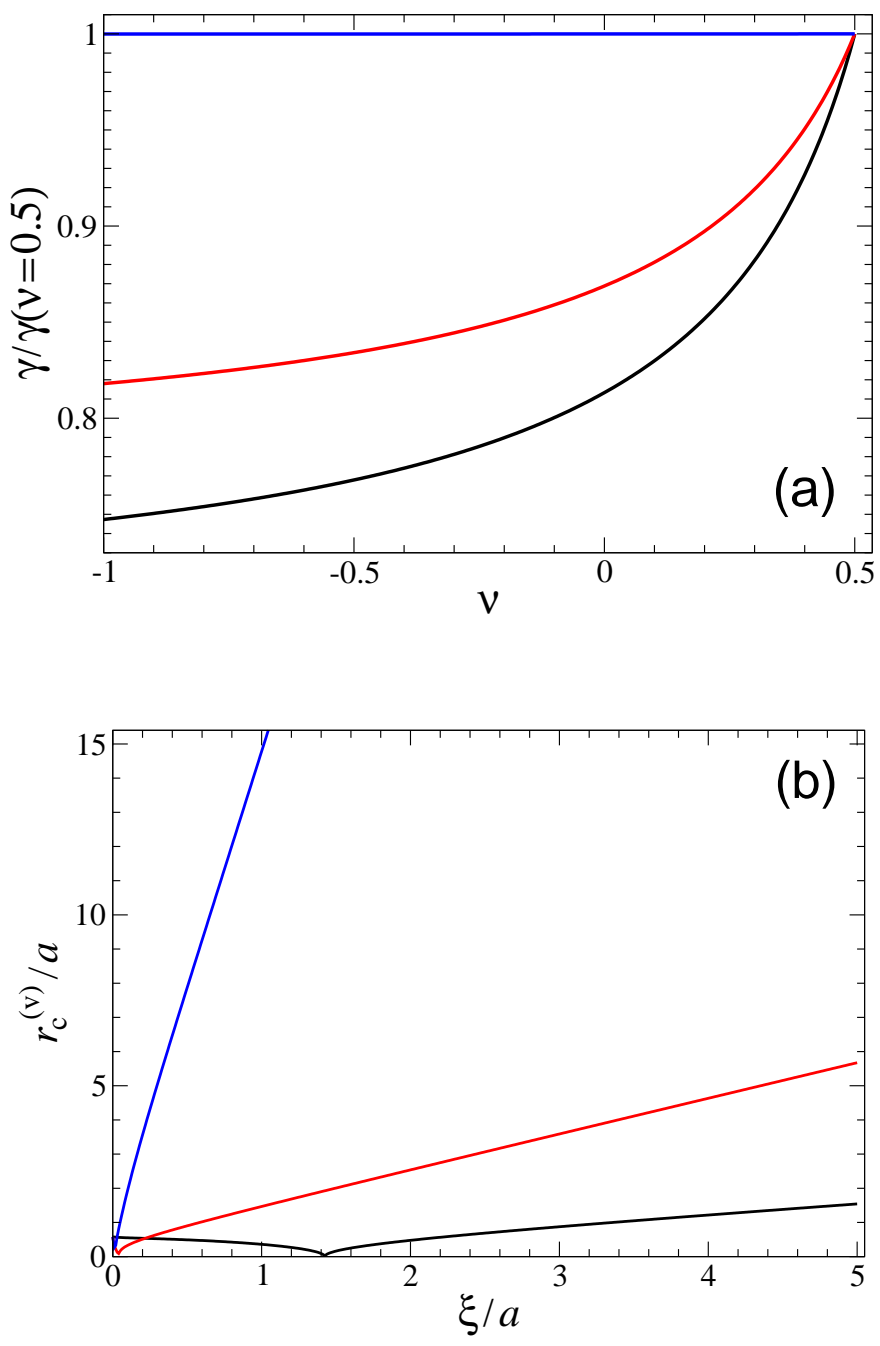

Fig. 2. (a) Friction coefficient, scaled by its value for an incompressible network, as a function of network Poisson ratio. (b) Observable crossover distance as a function of correlation length, both scaled by the bead's radius. In both panels the three curves, from bottom to top, correspond to the three boundary conditions of sticking, slipping, and free network, respectively. The free-network case is practically independent of network compressibility (a) and characterized by a large crossover distance (b). Parameters: $\eta_{\mathrm{b}} / \eta=100, \xi / a=1$ (a), $\nu=0.45(\mathrm{~b})$.

beads [4,8]. It may be related also to the unexpectedly deep penetration of inert beads into actin networks [4.

In addition to the insensitivity of $\mathbf{v}^{(0)}$ to network compressibility, under the free-network boundary conditions the one-point $\gamma$ is also practically independent of compressibility [fig. 2(a)]. We are led to the conclusion that measuring this parameter for biopolymer networks using inert beads is probably impossible. 
One should be able to reproduce the results obtained above for the case of free-network boundary conditions by introducing local heterogeneity. For example, extending the work of refs. 10,11, one can consider a finite shell of solvent surrounding the bead and apply the twofluid model outside the shell. This, however, adds another length parameter to the model, which does not seem necessary to account for experiments.

The physical origin of the "phantom network" condition - the lack of contact between a semiflexible network and a bead larger than its mesh size - remains unclear. A depletion layer resulting from sphere-network repulsion, be it a result of a genuine interaction potential or an entropy-induced effect, would not prevent direct exchange of stresses over the experimentally relevant time scales. For dilute semiflexible networks that do not bind to the beads these stresses are apparently inconsequential.

\subsection{The intermediate dynamic length}

The entire analysis highlights the key role played by an intermediate dynamic length, $\ell_{\mathrm{c}}(\omega) \equiv\left[\eta_{\mathrm{b}}(\omega) / \eta\right]^{1 / 2} \xi$, which was first pointed out in ref. 12. It is the crossover distance separating the intermediate, relative (Brinkman) flow from the asymptotic, collective (Stokes) flow. Unless the frequency is very high such that $\eta_{\mathrm{b}}(\omega) \simeq \eta, \ell_{\mathrm{c}}$ is much larger than the mesh size $\xi$. This length affects various dynamic properties. For example, for the GSR to hold, we have found that the bead must be larger than $\ell_{\mathrm{c}}$, not $\xi$. This is true for all boundary conditions studied, even in the case of a sticking network. (The bead should be even larger, $a \gg \ell_{c}^{2} / \xi$ in the cases of slipping or free networks.) This underlines again the problem in using one-point microrheology based on the GSR or GSER. The distance $\ell_{\mathrm{c}}$, although always affecting the crossover between the relative and collective flows, may be masked by a mixture of terms when the crossover distance of the observable flow, $r_{\mathrm{c}}^{(v)}$, is measured. However, in the case of free-network boundary conditions, as in actin networks, $r_{\mathrm{c}}^{(v)}$ is proportional to $\ell_{\mathrm{c}}$, pushing the observed crossover to a distance much larger than $\xi$, as demonstrated in fig. 2(b), and as confirmed experimentally [12,13].

\subsection{Further experimental consequences}

The scaling relations derived in sect. 4.4 suggest ways to sensitively extract various elusive parameters through extended microrheology combining one- and two-point measurements. For example, to get the network's Poisson ratio (which may be frequency-dependent), one should use a bead with surface groups that bind the network [4, 8 , to ensure stick boundary conditions, for which the sensitivity of $\gamma$ to $\nu$ is maximum [fig. 2(a)]. One may then apply the first relation in eq. (59) to extract $\nu$. Here, the one-point and two-point measurements are needed to get $\gamma$ and a reliable $\eta_{\mathrm{b}}(\omega)$, respectively. Another example is the ability to extract the network's correlation length and its dependence on parameters other than network concentration, using eq. (62). This has already been successfully demonstrated in refs. 12,13 .

\subsection{Open issues}

We conclude with three broader issues raised by the present study.

The first problem concerns a deficiency in the twofluid model. There is a certain inconsistency in the way the model is constructed. On the one hand, it should break down at length scales smaller than the mesh size $\xi$, where, obviously, the network cannot be treated as a viscoelastic continuum. On the other hand, one of the model parameters used at all scales is the solvent viscosity $\eta$, although it is physically meaningful only at scales smaller than $\xi$. In the case of the free-network boundary conditions (sect. 4.3), the model seems to correctly cover the entire range from $a / \xi \rightarrow \infty$ down to $a / \xi \rightarrow 0$; eq. (56) appropriately reproduces $\gamma=6 \pi \eta_{\mathrm{b}} a$ in the former limit, and $\gamma=6 \pi \eta a$ in the latter. The validity question can be posed in the following practical terms: can one extract the solvent viscosity by tracking the motion of a bead larger than $\xi$ ? If one believes the expressions derived in sect. 4, the answer is evidently positive; for example, to get $\eta$ one could fit one-point measurements in actin networks to eq. (56), or two-point measurements to eq. (58). In practice, attempts to do so yielded unreasonable results 27. At least in this restricted sense of the meaning of $\eta$, the failure of the model extends to lengths (e.g., particle sizes) much larger than $\xi$ and, therefore, is unrelated to the breakdown of the continuum limit. When using a combination of oneand two-point measurements, there is a way to bypass this problem, which was demonstrated to work well in the case of actin networks 12,13. As already mentioned in sect. 4.4, one can use the one-point measurement to define a local viscosity as sensed by the bead, $\eta_{l} \equiv \gamma /(6 \pi a)$, and substitute it for $\eta$ in the expressions relevant to twopoint measurements, such as $\mathbf{v}^{(1)}$ and $r_{\mathrm{c}}^{(u, v)}$. Since the onepoint property $\gamma$ depends on particle size and frequency, so does the local viscosity, $\eta_{l}=\eta_{l}(a / \xi, \omega)$, with the two limits $\eta_{l}(0, \omega)=\eta$ and $\eta(\infty, \omega)=\eta_{\mathrm{b}}(\omega)$. The necessity to define the length-scale-dependent $\eta_{l}$ indicates that an actual complex fluid is not fully characterized by uniform moduli as assumed by the two-fluid model. Instead, one should introduce wavevector-dependent viscosities.

The second issue has to do with the emergence of the mass-dipole term governing the relative, intermediate flow. The mass dipole in eq. (41), proportional to $\ell_{\mathrm{c}}^{2} / \eta_{\mathrm{b}}=\xi^{2} / \eta$ is independent of the particle size. This is curious, because a vanishingly small particle obviously cannot displace any fluid mass. The way in which an effective mass dipole builds up within a correlation "pore" of characteristic size $\xi$, and what happens when there are many such characteristic lengths, or none at all (as in a fractal structure), are interesting questions to be answered.

Finally, we would like to point out a possible relation between the dynamic length $\ell_{c}$ discussed here and the divergent dynamic length in polymer gelation and colloidal 
glass transitions (and, perhaps, amorphous solidification in general 28]). We notice that, as the material solidifies and $\eta_{\mathrm{b}}(\omega \rightarrow 0)$ diverges, so does $\ell_{\mathrm{c}}$. Within the description laid out here, the physics accompanying this transition is intuitively clear. As the crossover length $\ell_{c}(\omega \rightarrow 0)$ diverges, the intermediate, Brinkman region stretches out to infinity. The fluid, flowing relative to the network at all distances, loses its translational invariance, and the network turns into a solid porous matrix.

I am indebted to Adar Sonn-Segev and Yael Roichman for a fruitful collaboration and many discussions. Helpful discussions with Yitzhak Rabin and Tom Witten are gratefully acknowledged. This research was supported by the Israel Science Foundation under Grants No. 8/10 and No. 164/14.

\section{References}

1. T. M. Squires, T. G. Mason, Annu. Rev. Fluid Mech. 42, $413(2010)$

2. T. G. Mason, D. A. Weitz, Phys. Rev. Lett. 74, 1250 (1995).

3. J. C. Crocker, M. T. Valentine, E. R. Weeks, T. Gisler, P. D. Kaplan, A. G. Yodh, D. A. Weitz, Phys. Rev. Lett. 85, 888 (2000).

4. J. L. McGrath, J. H. Hartwig, S. C. Kuo, Biophys. J. 17, 3258 (2000).

5. D. T. Chen, E. R. Weeks, J. C. Crocker, M. F. Islam, R. Verma, J. Gruber, A. J. Levine, T. C. Lubensky, A. G. Yodh, Phys. Rev. Lett. 90, 108301 (2003).

6. M. L. Gardel, M. T. Valentine, J. C. Crocker, A. R. Bausch, D. A. Weitz, Phys. Rev. Lett. 91, 158302 (2003).

7. L. Starrs, P. Bartlett, Faraday Discuss. 123, 323 (2003).

8. M. T. Valentine, Z. E. Perlman, M. L. Gardel, J. H. Shin, P. Matsudaira, T. J. Mitchison, D. A. Weitz, Biophys. J. 86 4004 (2004).

9. H. C. Fu, V. B. Shenoy, T. R. Powers, Phys. Rev. E 78, 061503 (2008)

10. A. J. Levine, T. C. Lubensky, Phys. Rev. Lett. 85, 1774 (2000).

11. A. J. Levine, T. C. Lubensky, Phys. Rev. E 65, 011501 (2001).

12. A. Sonn-Segev, A. Bernheim-Groswasser, H. Diamant, Y. Roichman, Phys. Rev. Lett. 112, 088301 (2014).

13. A. Sonn-Segev, A. Bernheim-Groswasser, Y. Roichman, Soft Matter 10, 8324 (2014).

14. P.-G. de Gennes, Macromolecules 9, 587 (1976).

15. P.-G. de Gennes, Macromolecules 9, 594 (1976).

16. M. Doi, A. Onuki, J. Phys. II France 2, 1631(1992).

17. S. T. Milner, Phys. Rev. E 48, 3674 (1993).

18. A. J. Levine, T. C. Lubensky, Phys. Rev. E 63, 041510 (2001).

19. R. Bruinsma, A. Y. Grosberg, Y. Rabin, A. Zidovska, Biophys. J. 106, 1871 (2014).

20. H. C. Brinkman, Appl. Sci. Res. A1, 27 (1947).

21. D. Long, A. Ajdari, Eur. Phys. J. E 4, 29 (2001).

22. H. Diamant, Isr. J. Chem. 47, 225 (2007).

23. H. Diamant, J. Phys. Soc. Jpn. 78, 041002 (2009).

24. See Mathematica(C) and PDF files provided on-line as supplementary material.

25. V. Pelletier, N. Gal, P. Fournier, M. L. Kilfoil, Phys. Rev. Lett. 102, 188303 (2009).
26. F. C. MacKintosh, A. J. Levine, Phys. Rev. Lett. 100, 018104 (2008).

27. A. Sonn-Segev, Y. Roichman, private communication. 28. H. Diamant, arXiv:1406.2508 